\title{
Die EU-Politik der britischen Koalitionsregierung: Distanz vor Pragmatismus
}

\author{
David Allen*
}

Bei den Wahlen im Mai 2010 gelang es den Briten erstmalig nicht, die jahrzehntelange Tradition aufrechtzuerhalten und eine der politischen Parteien mit eindeutiger Mehrheit an die Regierung zu wählen. Nach fünf Tagen intensiver Verhandlungen wurde schließlich eine Regierungskoalition aus Conservatives (Konservativen) und Liberal Democrats (Liberaldemokraten) gebildet - zwei Parteien mit sehr unterschiedlichen Ansichten über die Europäische Union und die britische Europapolitik. Premierminister der Koalitionsregierung wurde David Cameron, der Vorsitzende der Konservativen, zu seinem Stellvertreter wurde Nick Clegg benannt, der den Liberaldemokraten vorsteht. Formal gab es in Großbritannien seit der 1945 beendeten Kriegskoalition von Winston Churchill keine Koalitionsregierung mehr, auch wenn zwischen März 1977 und Mai 1979 die Callaghan-Regierung ihre Mehrheit verlor und nur durch eine Reihe von Ad hoc-Absprachen mit den Liberalen, den Ulster Unionisten und den schottischen und walisischen nationalistischen Parteien an der Macht bleiben konnte.

\section{David Cameron und die Europawahl 2009}

In seinem letzten Jahr als Premierminister veranlasste der Skandal um die Spesensünder im britischen Parlament Gordon Brown zu dem Versuch, die Aufmerksamkeit weg von der Innenpolitik hin zur weltweiten Wirtschafts- und Finanzkrise zu lenken. Im Frühjahr 2009 bezeichnete er die Europäische Union in seiner Rede vor dem Europäischen Parlament als hervorragend geeignet, um die Welt aus der wirtschaftlichen Rezession herauszuführen. Während der Vorbereitungen zum G20-Gipfel in London verkündete Brown, dass Großbritannien seinen globalen Einfluss über Brüssel stärken könnte. Er überraschte seine Zuhörer ganz entgegen seiner bisherigen Äußerungen über die Europäische Union - mit der Feststellung, dass er ,stolz darauf sei, Brite zu sein, und stolz darauf, Europäer zu sein,“ und dass der Platz Großbritanniens nicht im Windschatten Europas zu suchen ist, sondern es fest in dessen Kern verankert ist. Dies war wohl dem Versuch geschuldet, die G20-Verhandlungen durch eine gemeinsame europäische Position mit zu gestalten. Letztlich überwog jedoch das Gefühl, dass das G20-Treffen lediglich eine Tarnveranstaltung für einen G2-Gipfel der USA und China war, bei dem Großbritannien - mit oder ohne seine EU-Partner - kaum eine Rolle spielte. Brown scheiterte mit seinem Versuch, seine engsten EU-Partner von seinen Absichten zu überzeugen, die seine Nähe zu den USA mit Argwohn betrachteten. Ein weiteres Beispiel für Browns Anliegen, sich Gehör zu verschaffen und seine Hinwendung zur Europäischen Union zu bekräftigen, zeigte sich in seinem Vorschlag zur Reform der Europäischen Investitionsbank vom Mai 2009. ${ }^{1}$ Dies war ein seltenes Beispiel für eine Initiative zur Aufwertung europäischer Institutionen durch einen führenden britischen Politiker, selbst wenn dieser freilich dadurch von den Problemen im eigenen Land ablenken wollte. Der Versuch,

\footnotetext{
* Prof. David Allen, Professor of European and International Politics, University of Loughborough, Vereinigtes Königreich.

1 Jean Englesham: Brown tries to shift focus to Europe, in: Financial Times, 27.05.2009.
} 
eine britische Führungsrolle in der Europäischen Union zu initiieren, spielte zu Beginn der Bankenkrise eine bedeutende Rolle. Als sich die Krise zunehmend auf die Eurozone konzentrierte und die britischen Wahlen näher rückten, verlor diese Initiative eines Nichtmitglieds der Eurozone allerdings an Bedeutung.

Die Europawahlen vom Juni 2009 waren in Großbritannien ein unprätentiöses Ereignis. ${ }^{2}$ Die großen Parteien investierten nicht in die Wahlkampagnen und zogen es vor, ihre Energien für die nächsten nationalen Wahlen im Jahr 2010 aufzusparen. Als klarer Sieger der Europawahlen ging im Königreich die europaskeptische United Kingdom Independence Party (UKIP) hervor, die mit 16,5 Prozent der Stimmen einen größeren Stimmenanteil aufwies als die regierende Labour Party (Labour-Partei). Wie diese gewann sie 13 Abgeordnetensitze. Die Konservativen konnten ihre Position festigen und gewannen mit einem Stimmenanteil von 27,7 Prozent insgesamt 25 Abgeordnetensitze. Die pro-europäischen Liberaldemokraten kamen nur auf den vierten Platz und erzielten mit einem Stimmenanteil von 13,7 Prozent immerhin elf Abgeordnetensitze. Bei den kleineren Parteien war der Schock groß, als die rechtsgerichtete British National Party (BNP) 6,2 Prozent der Stimmen erhielt und zwei Abgeordnete nach Straßburg schickte. Die Scottish National Party (SNP) erhielt - wie auch die Grünen - zwei Abgeordnetensitze, die Welsh Nationalists (Plaid Cymru) einen Abgeordnetensitz. Die Ergebnisse waren eine weitere Niederlage für die Labour-Regierung, und das gute Abschneiden der UKIP weckte bei den Konservativen große Sorgen mit Blick auf die kommende britische Parlamentswahl. Diese fürchteten - wie sich dann herausstellen sollte unnötigerweise - um ihre Stimmen aus dem rechten Lager, da schon ein kleiner Zuwachs für die UKIP bei den nationalen Wahlen Stimmenverluste für die eigene Partei mit sich gebracht hätte. Bereits der Verlust weniger Wahlkreise hätte ausgereicht, die Siegeschancen der Konservativen erheblich zu schmälern. ${ }^{3}$

Cameron löste nach den Europawahlen sein Versprechen ein, das er bei seiner Wahl zum Parteivorsitzenden gegeben hatte: Er forcierte den Auszug der britischen Konservativen aus der damaligen Fraktion der Europäischen Volkspartei (Christdemokraten) und europäischen Demokraten (EVP) im Europäischen Parlament und formierte die neue anti-föderalistische Fraktion Europäische Konservative und Reformer, die heute die viertgrößte Gruppe im Europäischen Parlament stellt und 55 Europaparlamentarier aus acht Ländern beheimatet. 26 britische Abgeordnete der Konservativen, 15 polnische Abgeordnete der Partei Recht und Gerechtigkeit (Prawo i Sprawiedliwość, PiS), neun tschechische Abgeordnete der konservativen Demokratischen Bürgerpartei (Občanská demokratická strana, ODS) und fünf weitere Abgeordnete, je einen aus Belgien, Finnland, Litauen, Ungarn und den Niederlanden. Im Vorfeld der Wahlen 2010 ging mancher davon aus, dass Cameron sich der EVP wieder anschließen würde, sobald er zum Premierminister gewählt werden würde. Dies war jedoch nach den britischen Wahlen 2010 nicht der Fall, noch nicht einmal dann, als der Fraktionsvorsitzende Michał Kamiński mit der Erklärung zurücktrat, dass die Gruppe von Extremisten übernommen worden sei! Die andauernde Distanzierung von der EVP ist den Europaskeptikern unter den Konservativen geschuldet, die sich mit den föderalistischen Ideen in der EVP nicht anfreunden können.

Der Weggang der Konservativen aus der EVP und die Neugründung der Fraktion Europäische Konservative und Reformer provozierten empörte Reaktionen der deutschen Bun-

2 Zu den Ergebnissen der Europawahl 2009 in Großbritannien siehe die Zusammenstellung des UK Office of the European Parliament: Results of 2009 European elections in the UK, 17.07.2011, abrufbar unter: http://www. europarl.org.uk/section/european-elections/results-2009-european-elections-uk (letzter Zugriff: 25.10.2011).

3 Gaby Hinsliff/Henry McDonald: UKIP threat to a Cameron majority, in: The Observer, 25.10.2009. 
deskanzlerin und des französischen Präsidenten. Beide fürchteten einen Machtverlust der EVP, sowohl im Europäischen Parlament als auch bei den Vorbereitungen der Gipfeltreffen des Europäischen Rates. Obwohl sich seit der Wahl die Beziehungen zwischen Cameron und den deutschen und französischen Spitzenpolitikern verbessert haben, zeigt der Entschluss der Konservativen, der EVP fern zu bleiben, doch sehr eindeutig den Einfluss des europaskeptischen Parteiflügels - und damit auch den Willen des Parteivorsitzenden bloß nicht den Anschein zu erwecken, weitere EU-Integrationsschritte mit britischer Beteiligung zu unterstützen.

Auch außerhalb der Europäischen Union wurde der Entschluss der britischen Konservativen, die EVP zu verlassen, nicht mit Wohlwollen aufgenommen. Den Vereinigten Staaten von Amerika musste William Hague, damals Schattenaußenminister, versichern, dass die neuen Verbündeten der Konservativen im Europäischen Parlament Opfer einer Schmierenkampagne der pro-europäischen britischen Presse, insbesondere des Guardian, geworden waren. Dies hielt jüdische Interessengruppen in den USA allerdings nicht davon ab, ihren Unmut über Kamiński von der PiS zu äußern, dem sie antisemitisches Verhalten vorwarfen. Als Hague der US-Außenministerin Hillary Clinton einen Besuch abstattete, trug auch sie ihre Bedenken vor und wiederholte das US-Mantra, das viele britische Politiker nicht hören wollen: Den USA ist an einer vollständigen Integration Großbritanniens in die Europäische Union gelegen, da somit im Ermessen der USA auch die Einflussmöglichkeiten Großbritanniens steigen. Die US-Kritik an der Entscheidung der Tories, die EVP zu verlassen, hatte bereits Tradition und ist nicht nur der heutigen Obama-Administration zu eigen. ${ }^{4}$

Die Europäische Union war wieder Thema auf dem Parteitag der Konservativen im Oktober 2009. Der beliebte rechtsgerichtete Bürgermeister von London, Boris Johnson, der von einigen als möglicher Nachfolger Camerons gehandelt wurde, geriet mit Kenneth Clarke, dem ehemaligen Finanzminister der Konservativen, über die Frage einer künftigen Stärkung der EU-Finanzmarktregulierung aneinander. Johnson versprach, die Europäische Kommission wegen ihres Vorhabens zu bekämpfen, die City of London regulieren zu wollen. Woraufhin Clarke erwiderte, dass weder Großbritannien noch die USA in der Position seien, den Franzosen und Deutschen Lektionen zu erteilen, nach dem ,katastrophalen Versagen“ der Finanzmarktregulierung, das diese Krise erst verursacht hat. Als schließlich die Konservativen durch die Koalition an die Macht kamen, wurden das Thema der EU-Finanzmarktregulierung und die allgemeine Ausrichtung der britischen Europapolitik zu einer parteiinternen Angelegenheit, die sich auf die EU-Politik der ganzen Koalition auswirken sollte.

Diesen beschämenden Streitpunkt der Tories machte sich die Labour-Partei zunutze, indem sie die Europapolitik wieder zum Gegenstand der Debatten machte. David Miliband, der damalige Außenminister, ließ den Streit über die EVP-Entscheidung wieder aufleben. In einem Artikel für The Observer wies er darauf hin, dass die neuen Verbündeten der Tories im Europäischen Parlament - sollten sie gewählt werden und weiterhin mit diesen rechtsgerichteten Parteien verbunden bleiben - dem Ruf Großbritanniens im Ausland schwer schaden könnten. Seiner Ansicht nach würde es ,auf Skepsis in Peking und Delhi stoßen, und erst recht in Berlin und Paris, wenn sich eine Partei, die die Regierungsmacht in Großbritannien anstrebt - noch dazu die Partei Churchills -, für solche Verbündete entscheidet" .5

4 Bereits 2006 hatte der damalige republikanische Präsidentschaftskandidat John McCain sein Unbehagen über einen Rückzug der Tories aus der EVP in einem Interview mit der Financial Times bekundet. Er verlieh seiner Hoffnung Ausdruck, dass die britischen Konservativen doch die Unterstützung der EVP anerkennen mögen, die sie ihnen zuteilwerden ließ, als sie allein auf weiter Flur standen.

5 Toby Helm/Rajeev Syal: Miliband in fresh attack on Tory links to far right, in: The Observer, 11.10.2009. 


\section{David Cameron und der Vertrag von Lissabon}

Der Vertrag von Lissabon konnte schließlich am 1. Dezember 2009 in Kraft treten, nachdem die Iren in einem zweiten Referendum für den Vertrag gestimmt hatten und der tschechische Präsident Václav Klaus seine Unterschrift nicht länger verweigerte. ${ }^{6}$ Cameron hatte seine anti-europäische Haltung erneut mit seiner Intervention in letzter Minute in Sachen Vertrag von Lissabon bewiesen, als er in einem handschriftlichen Brief den tschechischen Präsidenten bedrängte, seiner Haltung treu zu bleiben und den Vertrag nicht zu ratifizieren. Dieser letzte Versuch, den Vertrag von Lissabon noch zu boykottieren, hatte entschiedene Worte der Staats- und Regierungschefs aus Frankreich, Deutschland und Spanien auf dem Oktobergipfel des Europäischen Rates hervorgerufen. Brown, darauf bedacht, die Feindseligkeiten seiner europäischen Amtskollegen gegen Cameron zu nutzen, wählte das Gipfeltreffen, um seiner eigenen Kritik an der Europapolitik von Cameron Gehör zu verschaffen und ließ verlautbaren, dass „die Konservativen außerhalb des europäischen Mainstreams“ stünden.

Cameron reagierte darauf mit einer Mischung aus Pragmatismus und Mut, als er ankündigte, dass er - sollte er die Wahlen gewinnen - kein Referendum zum Vertrag von Lissabon abhalten lassen würde, jetzt wo dieser ratifiziert und bis zu seinem Amtsantritt bereits in Kraft getreten sein würde. Obwohl die ersten Reaktionen innerhalb der Konservativen Partei zurückhaltend waren, bestand im Rest Europas die Befürchtung, dass diese Stimmung wieder umschlagen könnte, und dass sich die Konservativen nach einem Wahlsieg als Regierung wieder von der Europäischen Union abwenden könnten. Anzeichen dafür lieferten sowohl der verhältnismäßig große Einfluss des rechten Flügels in der Partei und die fortbestehenden Vorbehalte in der Öffentlichkeit zur britischen EU-Mitgliedschaft. Im September 2009 ergab eine „You Gov“-Umfrage, dass - hätten sie die Wahl - nur 23 Prozent der Befragten sich für den Vertrag von Lissabon und gegen einen EU-Austritt entscheiden würden, während 31 Prozent der Befragten sich zu dieser Frage keine abschließende Meinung gebildet hatten und 43 Prozent sich für den Austritt aus der Europäischen Union entscheiden würden. ${ }^{7}$ Der Wahlerfolg der Konservativen hing demnach auch von der Unterstützung der Wähler für UKIP und BNP ab und hätte den Konservativen letztlich den Sieg kosten können.

Trotz ihrer Entscheidung, kein Referendum zum Vertrag von Lissabon zu initiieren, lehnten die Tories weiterhin die Stärkung der EU-Finanzmarktregulierung und das EU-Auslieferungssystem entschieden ab und lösten damit Alarmsignale bei den britischen Polizeichefs und den Strafverfolgungsbehörden aus. Auch wenn der europäische Haftbefehl letztlich akzeptiert wurde, blieb es dabei, dass die Tories alles in ihrer Macht Stehende unternehmen würden, weitere Kooperationsformen und Kompetenztransfers im Polizeibereich zu verhindern. Außerdem kündigte Cameron an, dass er die Auswirkungen des Vertrags von Lissabon abzufedern versuchen würde, indem er die Rückführung einiger Befugnisse verhandeln und an den Vorschlägen zu einem Sovereignty Act festhalten würde, der weitere Kompetenztransfers Richtung Brüssel verhindern soll. Um dies zu gewährleisten, kündigte er ebenfalls an, dass unter seiner Regierung jeder künftige EU-Vertrag einem Referendum unterzogen werden würde. Ein einfaches Versprechen in Anbetracht der Tatsache, dass weitere EU-Verträge zunächst einmal unwahrscheinlich zu sein schienen. Schon heute ermöglicht es der Vertrag von Lissabon Großbritannien zu entscheiden, ob und in welchem Maß es an EU-Ak-

6 Bereits im Vorfeld begann sowohl die Lobbyarbeit für den neu geschaffenen Posten des Präsidenten des Europäischen Rates als auch für die neue Europäische Kommission, die Anfang 2010 ihre Arbeit aufnehmen sollte.

7 Max Hastings: Europe is still a looming crisis for Cameron, in: Financial Times, 17.11.2009. 
tivitäten in den Bereichen Justiz, Polizei und Migrationskontrolle teilnehmen möchte, wie dies auch durch die Ausnahmeregelung zur Charta der Grundrechte der Europäischen Union (EU-Grundrechtecharta) der Fall ist. Cameron kündigte an, die EU-Grundrechtecharta dennoch grundsätzlich nicht mittragen zu wollen. Dabei erhielt er - wohl ungewollt - Unterstützung vom französischen Europaminister Pierre Lellouche, der diese Haltung zunächst bissig kommentierte und als ,erbärmlich“, ,autistisch“ und sogar als „,kastriert“ bezeichnete, ${ }^{8}$ bevor er den britischen Botschafter in Frankreich anrief, um ihm zu erklären, dass seine Kommentare falsch übersetzt worden seien und dass er wirklich nur sagen wollte, dass er die Politik von Cameron „,traurig“ finde. Zur gleichen Zeit nahm der französische Präsident hingegen mit besonders viel Wohlwollen zur Kenntnis, dass Cameron nach seiner möglichen Wahl nicht an dem Vorhaben eines Referendums zum Vertrag von Lissabon festhalten würde.

Gleich nach Ratifizierung des Vertrags von Lissabon begannen in der Europäischen Union die Verhandlungen über die Ernennungen des Präsidenten des Europäischen Rates sowie des Hohen Vertreters der Union für Außen- und Sicherheitspolitik und Vizepräsidenten der Europäischen Kommission (Hoher Vertreter). Der Schachzug der britischen Regierung, Tony Blair für den Posten als ständiger Präsident des Europäischen Rates ins Rennen zu schicken, fand bei den Konservativen keinen Anklang. Hague begründete dies damit, dass die Europäische Union keine „so Rampenlicht hungrige Persönlichkeit“" wie Blair nominieren sollte, die den Staats- und Regierungschefs nur die Fäden aus der Hand nehmen würde. Hague stellte klar, dass die Konservativen einen „Vorsitzenden und keinen Boss“ vorziehen würden, wie es mit Herman Van Rompuy dann auch der Fall war. Die Konservativen hielten auch nicht allzu viel von dem britischen Vorhaben, entweder den Posten des Präsidenten des Europäischen Rates oder des Hohen Vertreters für einen Briten zu beanspruchen. Sie forderten stattdessen, dass Großbritannien sich für eines der wichtigen Wirtschaftsressorts der neuen Europäischen Kommission - wie etwa Handel, Binnenmarkt, Wettbewerb oder Haushalt - stark machen solle.

Letzten Endes entschied bei der Vergabe der beiden Spitzenpositionen ein komplexes Zusammenspiel unterschiedlichster Beweggründe, bei dem mitgliedstaatliche Rivalitäten und die Notwendigkeit politischer Ausgewogenheit maßgeblich waren. ${ }^{9}$ Als jedoch feststand, dass Van Rompuy (männlich, konservativ, aus einem kleinen Mitgliedstaat) Präsident des Europäischen Rates werden sollte, war es der unausweichlichen EU-Logik geschuldet, dass die Hohe Vertreterin die gegenteiligen Eigenschaften aufweisen sollte (weiblich, eher linksgerichtet und aus einem großen Mitgliedstaat). Nach der Nominierung Catherine Ashtons als Hohe Vertreterin und Vizepräsidentin der Europäischen Kommission lag es auf der Hand, dass keiner der bedeutenden wirtschaftlichen Posten in der Kommission an einen britischen Kandidaten vergeben werden würde. Die Nominierungen der beiden EU-Spitzenposten wurden im In- und Ausland, wenn überhaupt, nur mit verhaltener Begeisterung aufgenommen. ${ }^{10}$ Sie galten auch als Zeichen für eine zunehmende Einflussnahme der großen Mitgliedstaaten in der Europäischen Union, stets ängstlich darauf bedacht, ihre staatliche Autonomie zu schützen. ${ }^{11}$ Die Streitigkeiten über die Benennung von Ashton nahmen sogar ein solches Ausmaß an, dass sich Brown und Nicolas Sarkozy trafen, um die Wogen wieder

8 Nicholas Watt/Patrick Wintour/Allegra Stratton Hastings: France: autistic Tories castrated UK, in: The Guardian, 05.11.2009.

9 Tony Barber: The Appointments of Herman van Rompuy and Catherine Ashton, in: The JCMS Annual Review of the European Union in 2009, Journal of Common Market Studies, Annual Review 2010, S. 55-67; David Allen/Michael Smith: Relations with the Rest of the World, in: The JCMS Annual Review of the European Union in 2009, Journal of Common Market Studies, Annual Review 2010, S. 209-230.

10 Quentin Peel: US underwhelmed by Brussels appointments, in: Financial Times, 23.11.2009.

11 George Parker: Supremacy of the nation state wins out, in: Financial Times, 20.11.2009. 
zu glätten. Sarkozy hielt sich jedoch nicht zurück, ein Treffen auch mit denjenigen Mitgliedstaaten (offenkundig ohne Großbritannien) einzuberufen, die auf den Erhalt der Gemeinsamen Agrarpolitik bedacht waren. Dort prahlte er schließlich mit seinem Erfolg bei der Besetzung der Spitzenpositionen in der Europäischen Kommission, mit der das angelsächsische Wirtschaftsmodell in der Europäischen Union kaum Chancen haben dürfte.

\section{Das Thema Europäische Union im britischen Wahlkampf}

Mit Beginn des Jahres 2010 liefen in Großbritannien die Vorbereitungen für die kommenden nationalen Wahlen an. Insbesondere Cameron sah sich vor einer heiklen Gratwanderung: Er musste den anti-europäischen rechten Flügel seiner eigenen Partei im Zaum halten und gleichzeitig die britische Öffentlichkeit wie auch seine EU-Nachbarn davon überzeugen, dass er aus Großbritannien keinen renitenten oder unkooperativen Partner machen würde. Um seine rechts stehenden Verbündeten in Europa zu beschwichtigen, sandte er Nick Herbert, seinen Schattenumweltminister, nach Polen, um dort die PiS von der Mäßigung ihrer Ansichten über Homosexualität zu überzeugen, da er in dieser Frage nicht von Liberaldemokraten und der Labour-Partei vorgeführt werden wollte. Vermutlich auch auf Anweisung Camerons haben Hague und Schattenfinanzminister George Osborne ihre Haltung zur Europäischen Union gemäßigt, als der Wahltag näher rückte und der deutliche Vorsprung der Konservativen zu schrumpfen begann - und sich somit die Wahrscheinlichkeit eines sogenannten „hung parliament“, eines Patts im Parlament, deutlich abzeichnete.

Die Tories befanden sich in einer Zwickmühle und hätten es am liebsten vermieden, sich über die Europäische Union überhaupt zu äußern. Sie wollten sich weder der Kritik anderer Parteien aussetzen, noch sich den eigenen parteiinternen und kontrovers diskutierten Streitlinien rund um EU-Themen stellen. Grundsätzlich fürchteten die Tories, insbesondere wegen der Entscheidung Camerons, den Vertrag von Lissabon zu akzeptieren und kein Referendum abzuhalten, Stimmen an die UKIP zu verlieren. Die Konservativen besannen sich wieder auf ihre pragmatisch ausgerichtete EU-Politik, stets darauf bedacht, keine Grundsatzpolitik zu verfolgen, sondern von Fall zu Fall zu entscheiden und etliche Ausnahmeregelungen zum Vertrag von Lissabon mit ihren EU-Partnern zu verhandeln. Gleichzeitig kritisierten sie die UKIP für die traurige Bilanz ihrer Europaabgeordneten. Sogar der besonders anti-europäisch eingestellte Schattenverteidigungsminister Liam Fox sprach von einer anglo-französischen „Kooperation“ und Hague verkündete die „strategische Entscheidung“ der Konservativen, keinen Streit mit der Europäischen Union führen zu wollen. Er sprach auch dem Europäischen Auswärtigen Dienst seine Unterstützung zu und bekräftigte, dass er, sollte er Außenminister werden, sicherstellen würde, dass Großbritannien seine besten Diplomaten dem Europäischen Auswärtigen Dienst zur Verfügung stellen wird (allerdings sagte er nicht für wie lange und mit welcher Mission!). Hague ließ aber auch verlautbaren, dass sich Großbritannien unter einer konservativen Regierung aus der Europäischen Verteidigungsagentur zurückziehen würde und er jeden Versuch zu verhindern wüsste, einen Europäischen Währungsfonds zu etablieren oder dem Vereinigten Königreich den Euro aufzuzwingen.

Im Vorfeld der Wahlen sahen sich die Konservativen mit dem Problem konfrontiert, dass sie sich aus Angst, der UKIP Tür und Tor zu öffnen, den liberaldemokratischen Positionen nicht allzu sehr annähern konnten, während sie gleichzeitig versuchen mussten, sich der anscheinend wachsenden Wählerschaft der Liberaldemokraten möglichst attraktiv zu präsentieren. ${ }^{12}$ Als die Wahlen näher rückten, setzte sich in Großbritannien die Erkenntnis durch,

12 Hinsliff/McDonald: UKIP threat to a Cameron majority, 2009. 
dass Brown in der Europäischen Union nicht als Retter in der Not wahrgenommen wurde acht von zehn der Befragten in Frankreich, Deutschland, Italien und Spanien waren der Meinung, dass der Einfluss Browns unbedeutend oder sogar negativ sei. Dies war vermutlich der Tatsache geschuldet, dass die Bedeutung des Vereinigten Königreichs in der aufkommenden Eurokrise zu vernachlässigen war. Die mangelnde Begeisterung, die Brown in der Europäischen Union entgegen gebracht wurde, und sein fehlender Draht zur Obama-Administration erschwerten es ihm, sich im Wahlkampf als erfahrener und einflussreicher Staatsmann zu inszenieren. Clegg machte die eindeutig bessere Figur in der ersten TV-Debatte zur Außenpolitik und die Labour-Regierung wurde ausgerechnet zu dieser Zeit von der Europäischen Kommission aufgrund des hohen Haushaltsdefizits verwarnt. ${ }^{13}$

\section{Die Koalitionsvereinbarung 2010 - Eckpunkte der britischen Europapolitik}

Die Labour-Partei fuhr in den nationalen Wahlen 2010 eine herbe Wahlniederlage ein - allerdings verpassten auch die Tories die Regierungsmehrheit. Es folgte eine Woche der Ungewissheit, in der die drei großen Parteien versuchten, eine gangbare Koalition auf die Beine zu stellen. Schließlich gelang es Cameron, Clegg und dessen Liberaldemokraten davon zu überzeugen, mit ihm zu koalieren. Es folgten weitere Verhandlungen zu einer ganzen Reihe von Themen in denen die Koalitionspartner unterschiedliche Positionen vertraten - von denen nicht zuletzt etliche die Europapolitik betrafen -, um diese miteinander in Einklang zu bringen. Die Koalitionsvereinbarung, ${ }^{14}$ die schließlich Cameron als Premierminister und Clegg als seinen Stellvertreter vorsah, war eindeutig mehr als nur eine vorübergehende Maßnahme. Beide Parteien verpflichteten sich zu einer gemeinsamen vollen Legislaturperiode von ganzen fünf Jahren. Die Koalitionsvereinbarung enthält unter anderem die folgenden Punkte:

- Großbritannien soll eine grundsätzlich bejahende EU-Mitgliedschaft mit dem Ziel sicherstellen, dass ,alle Nationen Europas in der Lage sind, den Herausforderungen des globalen Wettbewerbs, der globalen Klimaerwärmung und der globalen Armut entgegenzutreten“.

- Während der Legislaturperiode des britischen Parlaments sollen keine weiteren Souveränitätstransfers möglich sein.

- Die bestehende Kompetenzverteilung in der Europäischen Union soll überprüft werden. Insbesondere wird sich die Koalition dafür einsetzen, die Anwendbarkeit der Arbeitszeitrichtlinie in Großbritannien zu begrenzen.

- Das Gesetz zur Mitgliedschaft in den Europäischen Gemeinschaften von 1972 ${ }^{15}$ (European Communities Act 1972) soll novelliert werden, um sicherzustellen, dass jede künftige Vertragsänderung, die einen Macht- oder Kompetenztransfer zur Europäischen Union vorsieht, von einem erfolgreichen Referendum mitgetragen wird - ein sogenannter „Referendumsriegel“", der auch für die Anwendung der Passerelleklauseln im Primärrecht gilt.

- Die Koalition wird die Angelegenheit einer Sovereignty Bill prüfen, um klarzustellen, dass die höchste Autorität in Sachen EU-Mitgliedschaft dem britischen Parlament obliegt.

- Das Vereinigte Königreich soll während der Sitzungsperiode weder der Eurozone beitreten noch einen solchen Beitritt vorbereiten.

- Bei den anstehenden Verhandlungen zum EU-Haushalt sollen die britischen Interessen vehement vertreten werden und der EU-Haushalt soll sich in erster Linie auf Bereiche konzentrieren, bei denen die Zusammenarbeit auf EU-Ebene einen „Mehrwert“ darstellt.

13 Larry Elliot/Patrick Wintour/Julian Glover: Blow for Brown as Europe warns UK to bring down debt, in: The Guardian, 16.03.2010.

14 The Guardian: The Coalition Agreement, 15.05.2010.

15 European Communities Act 1972, abrufbar unter: http://www.legislation.gov.uk/ukpga/1972/68/pdfs/ ukpga_19720068_en.pdf (letzter Zugriff: 25.10.2011). 
- Großbritannien soll Druck auf das Europäische Parlament ausüben, künftig nur noch in Brüssel zu tagen.

- Im Bereich des Strafrechts soll die künftige EU-Gesetzgebung von Fall zu Fall betrachtet werden, statt eher aus prinzipiellen Erwägungen heraus abgelehnt zu werden, wie es die erklärte Politik der Konservativen war.

- Schließlich soll die Koalition künftige Erweiterungen der Europäischen Union unterstützen. In der BBC wurde diese Vereinbarung beschrieben als ,,arrangierte Waffenruhe“ in EUAngelegenheiten zwischen den Konservativen und den Liberaldemokraten. Andere sahen darin lediglich kurzfristige Zugeständnisse an die Liberaldemokraten, während gleichzeitig die langfristigen Bestrebungen der Konservativen - eine möglichst distanzierte und eng begrenzte Beziehung Großbritanniens zur Europäischen Union - beibehalten wurden.

Nachdem die Koalitionsvereinbarung getroffen war, wählte Cameron Clegg aus, um die EUPartner zu umwerben. Clegg, der mehrere europäische Sprachen spricht, darunter Französisch, Deutsch und Spanisch, kam diesem Anliegen eifrig nach. Cameron ernannte außerdem den EUmoderaten David Lidington zum Europaminister, statt wie ursprünglich vorgesehen den europaskeptischen Mark Francois, der für den Rückzug der Konservativen aus der EVP verantwortlich war. Cameron passte seine leicht aggressive Haltung gegenüber der Europäischen Union den moderateren Tönen des neuen Koalitionspartners an. ${ }^{16}$ Die Liberaldemokraten ihrerseits gaben sich mit den gemäßigten Plänen der Tories zufrieden und unternahmen nur wenig, um ihre eigenen dezidiert pro-europäischen Ansichten durchzusetzen. Das Hauptanliegen von Clegg war es nicht, die EU-Beziehungen zu konsolidieren, sondern das britische Mehrheitswahlsystem zu ändern.

Mitte Juni 2010 nahm Cameron erstmals an einem Gipfeltreffen des Europäischen Rates teil. Da diesem Zusammentreffen bereits eine Reihe von erfolgreichen bilateralen Treffen mit hochrangigen europäischen Staatschefs vorausgegangen war, fiel der Empfang des britischen Regierungschefs und seiner Minister durch ihre EU-Kollegen entsprechend positiv und freundlich aus. Vermutlich waren diese erleichtert, dass sich ihre zumindest anfangs gehegten schlimmsten Befürchtungen über den Kurs der Europapolitik einer von Cameron geführten Regierung nicht bewahrheiteten. Im Lichte des britischen Wahlergebnisses und der Turbulenzen in der Eurozone schien ,die britische Politik europäischer und die europäische Politik britischer" ${ }^{\text {"17 }}$ geworden zu sein. Koalitionsbildung und Konsensfindung ersetzten, wenn auch nur vorübergehend die von Gegensätzen geprägte Politik in Großbritannien. Gleichzeitig machten sich europaskeptische Strömungen in den von der Eurokrise am stärksten in Mitleidenschaft gezogenen Mitgliedstaaten der Europäischen Union breit.

In seiner Rede im November 2009 - in der er erklärt hatte, dass er den Vertrag von Lissabon nicht aufkündigen würde - hatte Cameron seiner Partei in sechs ,Versprechen' seine Haltung in der Europapolitik versichert. Obwohl die Koalitionsregierung leichte Modifikationen bei den ersten drei vornahm, ${ }^{18}$ wurden alle Punkte in das umstrittene Europagesetz (den European Union Act) aufgenommen, das am 19. Juli 2011 in Kraft trat. ${ }^{19}$

16 Toby Helm: Secret hardline memo on Europe threatens Tory-Lib Dem coalition, in: The Observer, 08.05.2010.

17 Gideon Rachman: The fear that now unites Britain and Europe, in: Financial Times, 15.05.2010; siehe auch Timothy Garton Ash: Britain and Europe are living separate crises. Underneath it's the same one, in: The Guardian, 13.05.2010.

18 Diese Versprechen betreffen die Beteuerung, dass vielmehr das britische Parlament und nicht die Europäische Union souverän ist; das Referendumsversprechen bei allen künftigen EU-Vertragsänderungen, seien diese umfassend oder auch nur marginal; und die Zusicherung der Anwendung des britischen Primärrechts bei der Inkraftsetzung der Passarelleklauseln im europäischen Primärrecht.

19 Andrew Blick: The European Union Bill. A Federal Trust Briefing, London 2011; Federal Trust: The Coalition and Europe - After the Honeymoon. A Federal Trust Report, London 2011. 


\section{Der European Union Act 2011 - Spiegelbild britischer Europaskepsis}

Der European Union Act besteht aus drei wesentlichen Teilen. ${ }^{20}$ Einer ist praktischer Natur und enthält schlicht die britische Zustimmung zum Protokoll über die Übergangsbestimmungen des Vertrags von Lissabon, welches 18 zusätzlichen Abgeordneten ermöglicht, ihre Sitze noch vor den nächsten Wahlen 2014 im Europäischen Parlament einzunehmen. Der Großbritannien zustehende zusätzliche Abgeordnete soll aus dem Wahlkreis der Region der Midlands kommen.

Die bedeutsamen Teile des European Union Act sind hingegen die Bestimmungen, die ein Referendum in Großbritannien bei Änderungen der bestehenden EU-Verträge vorsehen. Ein solches wird nötig, bevor Großbritannien einem neuen Vertrag oder einer neuen Vertragsänderung zustimmen kann beziehungsweise für Entscheidungen, die auf Grundlage der bestehenden Rechtsgrundlage britische Machtbefugnisse oder Kompetenzen auf die Europäische Union „transferieren“ würden.

Zusätzlich enthält der European Union Act eine Souveränitätsklausel. ${ }^{21}$ Diese schafft eine Rechtsgrundlage für die seit Langem bestehende Praxis, EU-Recht in die britische Gesetzgebung durch den vom Parlament erlassenen European Communities Act 1972 zu übernehmen.

Der European Union Act bleibt durch und durch europaskeptisch, und das obwohl sich die Konservativen von ihrem vor der Wahl provokativ zur Schau getragenen Beharren auf einer Neuverhandlung der Bedingungen der britischen EU-Mitgliedschaft distanziert haben und die Liberalen ihre Forderungen nach einem sofortigen Referendum zu den Prinzipien der britischen EU-Mitgliedschaft fallen ließen. Er dient dazu, eine deutliche Grenze für den Platz des Vereinigten Königreichs im europäischen Integrationsprozess zu markieren. Für die geringsten Änderungen der EU-Verträge ist in Großbritannien ein Referendum oder ein vom Parlament verabschiedetes Gesetz erforderlich, während ein Rückzug Großbritanniens aus der Europäischen Union, zumindest theoretisch, ohne ein solches Referendum möglich ist.

Beim Gesetzgebungsverfahren zum European Union Act zeigte sich, dass lediglich aus dem House of Lords mit ernstzunehmendem Widerstand zu rechnen war, während ein zunehmend europaskeptisch eingestelltes House of Commons scheinbar begierig darauf wartete, künftigen Integrationsschritten durch ein obligatorisches Referendum einen Riegel vorzuschieben. Gegen einen solchen Riegel sprachen sich im House of Lords unter anderem die pro-europäischen Mitglieder früherer konservativer Regierungen aus, so wie Lord Howe, ehemaliger Finanzminister und Außenminister, dessen bittere Rücktrittserklärung 1990 zum Sturz von Margaret Thatcher führte. Die Ergänzungsvorschläge zum geplanten Europagesetz aus dem House of Lords scheiterten jedoch an der Regierung und im House of Commons. Versuche, für EU-Referenden (zumindest) eine Wahlbeteiligung von mindestens 40 Prozent und eine sogenannte Sunset-Klausel vorzusehen, die den „Integrationsriegel“ für den Fall, dass dieser nicht von einer künftigen Regierung erneuert werden würde, auf fünf Jahre beschränken sollte, wurden allesamt von Europaminister Lidington abgelehnt. Er begründete dies mit dem hohen $\mathrm{Maß}$ an Unzufriedenheit mit der Europäischen Union in der britischen Bevölkerung. Das Versprechen eines Referendums würde die Bedenken der Wähler ernst nehmen. Eigentlich hätte er aber auch sagen können, dass das Europagesetz dazu dient, die Europaskeptiker unter den Hinterbänklern der eigenen Partei zu beschwichtigen.

20 European Union Act, abrufbar unter: http://www.legislation.gov.uk/ukpga/2011/12/pdfs/ukpga_20110012_ en.pdf (letzter Zugriff: 25.10.2011).

21 Teil 3, Paragraph 18 European Union Act. 
Im House of Commons zeigte sich die wachsende Europaskepsis in der Abstimmung über die Erhöhung des britischen Beitrags zum Internationalen Währungsfonds (IWF). 246 Abgeordnete stimmten gegen eine solche Erhöhung - einfach aus dem Grund, dass der IWF am ,bail-out' der Eurozone beteiligt ist.

Der European Union Act war Teil der Koalitionsvereinbarung, obwohl dieses Schriftstück lediglich auf eine „Prüfung der Angelegenheit einer Sovereignty Bill“ verweist. Er wurde frühzeitig von den Konservativen vorangetrieben, um möglichen Zweifeln über den Einfluss der Liberaldemokraten auf die Europapolitik der Regierung den Wind aus den Segeln zu nehmen. Außenminister Hague damit auf dem Parteitag der Konservativen im Herbst 2010 nicht hinter dem Berg und bekam Standing Ovations für seinen nüchternen Ansatz gegenüber der Europäischen Union, als er sagte ,wir unterstützen wirksame Zusammenarbeit und entschlossenes Handeln, nicht aber eine Nabelschau und die Errichtung eines institutionellen Empires". Er führte weiter aus, dass sich die Europäische Union zu viel einmische und dass der Vertrag von Lissabon die institutionellen Rivalitäten sogar noch verschärft habe. Exemplarisch führte er dafür die Grabenkämpfe an, wie sie sich 2010 über der Errichtung des Europäischen Auswärtigen Dienstes entzündet hatten. Während sich Hague für den European Union Act einsetzte, war es Andrew Duff, ein führender Liberaldemokrat im Europäischen Parlament, der dagegen argumentierte, indem er diesen als „,kalkulierten Schachzug " beschrieb, der dazu dienen würde, eine nationalistische Wählerschaft anzusprechen und ,der die Befugnisse des britischen Parlaments in Europafragen schwächen würde, jetzt, wo der Vertrag von Lissabon den nationalen Parlamenten Gelegenheiten einräumt, sich konstruktiv in der EU zu beteiligen“. 22

\section{Weitere Wahlversprechen und ihre Realisierung in der Europäischen Union}

Was die anderen der von Cameron vor der Wahl gemachten Versprechen angeht, so hat die Regierung bisher noch keine ernsthaften Versuche unternommen, sich um Ausnahmeregelungen (,opt-outs') bei der EU-Grundrechtecharta oder in der europäischen Sozial- und Beschäftigungspolitik zu bemühen. Obwohl die Liberaldemokraten sich gerne damit schmücken würden, die Regierung bei diesen beiden Versprechen (die nicht in die Koalitionsvereinbarung aufgenommen wurden) in ihre Schranken gewiesen zu haben, sind es eher der Aufwand, der mit einer notwendigen Neuverhandlung verbunden wäre, und die Rücksichtnahme von Cameron auf die Befindlichkeiten der Liberaldemokraten, die den Premier bisher davon abhielten, entsprechende Schritte einzuleiten. Dennoch gibt es Stimmen in den eigenen Reihen, die auch diese beiden Punkte angesprochen sehen wollen, sobald über eine Vertragsrevision im Rahmen einer Stärkung der Finanzmarktregulierung in der Eurozone verhandelt wird.

Dem sechsten Versprechen von Cameron hat die Regierung Rechnung getragen, indem sie die Vorgaben des Vertrags von Lissabon dazu nutzte, bei bestimmten justiziellen strafrechtlichen Maßnahmen von den bestehenden Ausnahmeregelungen Gebrauch zu machen, und dies obwohl Großbritannien noch keine Entscheidung über ein ,opt-out ‘ zu Europol getroffen hat. Ende 2010 und im Einklang mit den Vorgaben der Koalitionsvereinbarung entschloss sich die Regierung bei sechs Maßnahmen aus dem Bereich des Raums der Freiheit, der Sicherheit und des Rechts mitzuwirken, bei fünf anderen verweigerte sie jedoch die $\mathrm{Zu}-$ sammenarbeit. Außerdem beteiligte sie sich an zwei Schengen-Maßnahmen. ${ }^{23}$ Eine der um-

22 Nicholas Watt: Liberal Democrats in coalition rift over sovereignty bill to curb EU, in: The Guardian, 12.11.2010.

23 Vaughne Miller: The Government's Policy on Europe, UK House of Commons Library: Standard Note, SN/ IA/5854, 07.02.2011, S. 26-27. 
stritteneren Maßnahmen, bei der sich die Regierung ebenfalls für ein ,opt-out' entschied, war eine EU-Richtlinie zur Koordinierung der Bemühungen im Bereich sexueller Menschenhandel und Zwangsprostitution. ${ }^{24}$ Dies wurde von der Opposition und, zumindest inoffiziell, auch von vielen Liberaldemokraten innerhalb der Koalitionsregierung verurteilt. Dem war die Enthüllung, dass die Zahl der Schuldsprüche für diese Verbrechen in Großbritannien schnell gesunken war, vorangegangen - für viele eine direkte Folge der unkoordinierten Maßnahmen der Europäischen Union gegen Aktivitäten, die häufig ihren Ursprung in Rumänien und Bulgarien haben.

Ironischerweise wird die generelle Zurückhaltung der Konservativen gegenüber einer Ausweitung von EU-Befugnissen im Bereich des Raums der Freiheit, der Sicherheit und des Rechts im Einzelfall aber auch durchbrochen, wie etwa der Vorschlag für den Aufbau eines EU-weiten Netzwerks von Passagiernamensregistern zeigt, der von der britischen Regierung begeistert aufgenommen wurde. Ein solches Netzwerk würde die Bewegungsabläufe und Personendaten aller Flugpassagiere in Europa registrieren und besteht bereits für Flüge zwischen der Europäischen Union und den USA, auch wenn es zurzeit im Europäischen Parlament unter Beschuss geraten ist. Die britische Innenministerin Theresa May führte eine Kampagne an, die im Ministerrat für eine EU-Richtlinie zur verpflichtenden Weitergabe von Passagierdaten warb. In Großbritannien erntete sie dafür harsche Kritik der Europaskeptiker aus der eigenen Partei sowie von Organisationen wie Open Europe. Der führende Europaskeptiker, der Abgeordnete Bill Cash, ${ }^{25}$ sprach von einer „Flut von EU opt-ins“. Die Gründe für eine Ausweitung der Passagiernamensregister auf den innereuropäischen Reiseverkehr sind eng verknüpft mit der britischen Haltung zu den Beziehungen der Europäischen Union mit den USA, da die Obama-Administration Druck auf die Europäische Union ausübt - insbesondere auf das Europäische Parlament -, den bereits bestehenden Datenaustausch auszuweiten. Dies ist ein Beispiel für einen Integrationsbereich, bei dem die Tories aus Prinzip dagegen sind, in der Praxis aber dafür!

Die Selbstverpflichtung, ein Referendum über jede EU-Vertragsrevision sowie einen künftigen Beitritt zur Eurozone abhalten zu wollen, führte dazu, dass das - zweite jemals in Großbritannien abgehaltene - Referendum am 5. Mai 2011 aufmerksam von EU-Beobachtern verfolgt wurde. Gegenstand war der von den Liberaldemokraten eingebrachte Vorschlag (ebenfalls in der Koalitionsvereinbarung enthalten), das Wahlsystem in Großbritannien vom Mehrheitswahlrecht zum Alternativstimmen-Wahlrecht zu ändern. ${ }^{26}$ Die Liberaldemokraten hatten ihre Niederlage von 1977-79 noch in bitterer Erinnerung, als sie versucht hatten, die Abhängigkeit der Labour-Partei von ihrer Zustimmung für sich selbst zu nutzen und das Verhältniswahlrecht bei den Wahlen zum Europäischen Parlament in Großbritannien durchzusetzen. Entsprechend hart kämpften sie 2011 gegen Konservative und Labour-Partei für ihren Vorschlag, mussten aber beim Referendum schließlich eine große Niederlage mit 68 Prozent Nein-Stimmen hinnehmen (bei 32 Prozent Ja-Stimmen und einer Wahlbeteiligung von 41 Prozent). Die Analyse des Referendums ist gerade im Hinblick auf die Selbstverpflichtung zu einem Referendum durch den European Union Act interessant. Zunächst einmal war die Wahlbeteiligung schwach und zudem schienen die meisten Wähler die Gelegenheit zu nutzen, ihre Unzufriedenheit mit den Liberaldemokraten zum Ausdruck

24 Erwägungsgrund 35 der Richtlinie 2011/36/EU des Europäischen Parlaments und des Rates vom 5. April 2011 zur Verhütung und Bekämpfung des Menschenhandels und zum Schutz seiner Opfer sowie zur Ersetzung des Rahmenbeschlusses 2002/629/JI des Rates, in: Amtsblatt der EU, Nr. L 101 vom 15. April 2011, S. 1-11.

25 Bill Cash ist Vorsitzender im EU-Untersuchungsausschuss des House of Commons.

26 John Curtice: The Death of a Miserable Little Compromise: The Alternative Vote Referendum, in: Political Insight 2/2011, S. 14-17. 
zu bringen, statt mit dem vorgeschlagenen konstitutionellen Wandel. Diese Unvorhersehbarkeit des Abstimmungsverhaltens bei Referenden könnte für künftige Regierungen eine schwierige Hürde darstellen, für einen Wandel in den europäisch-britischen Beziehungen zu werben.

\section{EU-Haushalt und Eurokrise aus britischer Sicht}

Ein großes Anliegen der neuen Regierung, das von Konservativen und Liberaldemokraten gemeinsam getragen wurde (und ebenfalls Gegenstand der Koalitionsvereinbarung ist), war der Wunsch das Volumen des EU-Haushalts $2011 \mathrm{zu}$ verringern sowie sicherzustellen, dass in dem nächsten mehrjährigen Finanzrahmen (2014-2021) die schwierige Haushaltslage aller Mitgliedstaaten berücksichtigt wird. Die Europäische Kommission verkündete ihren Ausgabenplan für den nächsten siebenjährigen Finanzrahmen trotz eines von Cameron im Dezember 2010 angeregten Briefs, der auch von den Regierungschefs aus Frankreich, Deutschland, den Niederlanden und Finnland unterzeichnet wurde und der dazu aufrief, die Erhöhung des EU-Haushalts bei dem nächsten mehrjährigen Finanzrahmen auf die aktuellen Ausgaben plus die Inflationsrate zu beschränken. ${ }^{27}$ Der Kommissionsvorschlag - so beanstandete die britische Regierung - stelle eine unakzeptable Erhöhung von zehn Prozent gegenüber dem vorherigen Finanzrahmen dar. Die politische Klasse in Großbritannien ,demonstrierte daraufhin, wie es selten vorkommt, politische Einigkeit, um gemeinsam den Vorschlag eines erstmals 1.000 Milliarden Euro teuren EU-Haushalt zu verurteilen“.28

Cameron unternahm mächtige Anstrengungen, um den Kommissionsvorschlag für den EU-Haushalt $2011 \mathrm{zu}$ begrenzen. Wieder suchte und erhielt er die Unterstützung anderer Mitgliedstaaten. Doch war ihm auch in diesem Fall kein großer Erfolg beschieden, auch wenn er behauptete, dass das Ergebnis besser ausfiel als erhofft. Bedeutsam bei den jährlichen und mehrjährigen Haushaltsentscheidungen ist, dass das Vereinigte Königreich mit seinen Forderungen nach Haushaltsbegrenzungen und -reformen nicht mehr alleine steht. Auch in den beiden Politikbereichen Gemeinsame Agrarpolitik und Strukturfonds, die den größten Anteil am EU-Haushalt ausmachen, ist die Gruppe der Kritiker heute größer, als es noch bei den Verhandlungen über die letzte Finanzielle Vorausschau (2007-2013) der Fall war. Die meisten Beobachter würden wohl sagen, dass die von Clegg unterstützte Politik Camerons in einer Linie steht mit der Politik seiner Vorgänger Blair und Brown. Zweifelsohne sieht sich die Europäische Kommission heute herausgefordert vom Vereinigten Königreich und den Nettozahlern unter den großen Mitgliedstaaten, wenn es darum geht, die Finanzplanung für die nächsten Jahre auf den Weg zu bringen.

Gemessen an den erforderlichen Mitteln zur Lösung der europäischen Bankenkrise und der Krise der Eurozone schätzen Wirtschaftswissenschaftler die Summen für das EU-Budget als verhältnismäßig gering ein und bewerten dieses daher als wirtschaftlich und finanzpolitisch irrelevant. Politisch spielen diese in der Europäischen Union hingegen eine umso größere Rolle - insbesondere im Vereinigten Königreich -, schließlich hängt die Gestaltung der Europapolitik der Mitgliedstaaten maßgeblich von der Innenpolitik und der öffentlichen Meinungsbildung ab. Es kann daher wohl kaum überraschen, dass sich Cameron öffentlich empörte, als Pläne für das neue, 300 Millionen Euro teure, Gebäude des Europäischen Rates bekannt wurden oder als die Details der hohen Ruhestandsregelungen für EU-Beschäftigte

27 Ursprünglich hatte Cameron eine substanzielle Reduzierung von 1,1 Prozent des Bruttoinlandsprodukts (BIP) der Europäischen Union auf 0,85 Prozent des BIP der Europäischen Union vorgeschlagen.

28 Nicholas Watt/Ian Traynor: Labour joins Tory attack on EU's $€$ 1tn budget proposal, in: The Guardian, 01.07.2011. 
veröffentlicht wurden. Wie so oft in der Vergangenheit waren die Versuche Großbritanniens, die Europäische Union wegen ihrer Ausgaben vorzuführen, wenig erfolgreich. Dies war beispielsweise der Fall, als die britische Regierung ihre Zustimmung zu einem Kommissionsvorschlag verweigerte, der einen Fonds für Flutopfer in osteuropäischen Mitgliedstaaten vorsah - dies mit dem Argument, dass damit zusätzliche Kosten verbunden seien und die Kommission diese durch Einsparungen an anderer Stelle abdecken sollte - ein weiteres Beispiel dafür, wie das Vereinigte Königreich eine wirtschaftliche Marginalie zu einem politischen Problem aufblähte.

Selbstverständlich sind die Summen, die für die Lösung der Eurokrise aufgewendet wurden - und weiterhin werden -, weitaus umfangreicher. Anfangs war die Cameron-Regierung entschlossen, ${ }^{29}$ sich aus der Eurozone herauszuhalten, unter anderem da eine britische Unterstützung der Rettungsfonds sie Wählerstimmen kosten dürfte. Dementsprechend findet sich die Cameron-Regierung heute mehr und mehr in der Rolle eines unerwarteten Fürsprechers einer Vertiefung der politischen und wirtschaftlichen Zusammenarbeit der Mitgliedstaaten der Eurozone wieder, um auf diese Weise den auch für Großbritannien wichtigen europäischen Markt zu erhalten und negative Folgen eines Zusammenbruchs des Euro zu vermeiden. Die Schwierigkeit dabei ist allerdings, dass sich die Regierung durch ihr Fernbleiben von den vorgeschlagenen Maßnahmen und der Diskussion über deren konkrete Ausgestaltung in einer denkbar ungünstigen Lage befindet: Einerseits muss sie befürchten, sich der Kritik der Europaskeptiker durch eine zu aktive Rolle bei den Rettungsplänen der Eurozone auszusetzen, und andererseits muss sie befürchten, dafür kritisiert zu werden, aus den Machtzirkeln - die sich mit dem Schicksal der Europäischen Union und möglicherweise sogar der globalen Wirtschaft beschäftigen - ausgeschlossen zu sein beziehungsweise in diesen nicht ausreichend gehört zu werden. Cameron scheint diese gegensätzlichen Pole mit einem Kompromiss in Einklang bringen zu wollen, indem er seine Führungskapazitäten dadurch unter Beweis stellt, den Regierungschefs der Eurozone zu sagen, wie sie zu handeln haben!

Sollten die Staaten der Eurozone sich schließlich auf einen Lösungsvorschlag einigen können, für den eine Vertragsänderung nötig werden sollte, dann wird sich die britische Regierung wahrscheinlich auf Druck ihrer Hinterbänkler gezwungen sehen, im Austausch für ihre Zustimmung zu einer Vertragsrevision auf Zugeständnisse bei der EU-Grundrechtecharta oder in der europäischen Sozial- und Beschäftigungspolitik zu pochen. Auch wenn die Zustimmung aller Mitgliedstaaten für eine Änderung des Vertragwerks unerlässlich ist, bleibt es doch fraglich, ob die britische Regierung tatsächlich einen solchen Einfluss ausüben kann. Ein solches Vorgehen, das in den Augen der britischen Europaskeptiker und vielleicht auch der Regierung selbst, als vorteilhafte Verhandlungsposition gelten dürfte, könnte sich allerdings auch nachteilig für das Vereinigte Königreich auswirken - wenn nämlich Großbritannien für das Scheitern eines Rettungspakets für die Eurozone verantwortlich gemacht werden würde.

\section{Britische Personalentscheidungen und ihre Folgen für den Stellenwert der EU}

Seit den Wahlen 2010 gab es in Großbritannien hinsichtlich der britischen Europapolitik einige interessante Ernennungen: Außenminister Hague, ein langjähriger Europaskeptiker, der 2001 die Wahlen in Großbritannien mit dem Schlachtruf „Rettet das britische Pfund“ als

29 Die Konservativen waren aus Prinzip und die Liberaldemokraten aus pragmatischeren Gründen entschlossen sich herauszuhalten, da zumindest in naher Zukunft keine Stimmen mit der Befürwortung des Euro zu gewinnen sind. 
Vorsitzender die Tories anführte (und verlor). Finanzminister Osborne ist wie Cameron nur mäßig enthusiastisch, wenn es um die Europäische Union geht. Diese haben aber in Justizminister Clarke ein Gegengewicht. Clarke, ein ehemaliger Finanzminister, ist einer der führenden Konservativen und favorisiert eine starke Rolle des Königreichs in der Europäischen Union. Als Zugeständnis an seinen Koalitionspartner ernannte Cameron einen Europabefürworter unter den Konservativen, Lidington, zum Europaminister. Dieser hat - anders als seine vielen Labour-Vorgänger - seinen Posten inzwischen mehr als ein Jahr inne. Interessanter ist die Berufung von Sir Jon Cunliffe als ersten Nichtdiplomaten auf den Posten des britischen Ständigen Vertreters bei der Europäischen Union. Cunliffe hat sich während seiner Zeit im Finanzministerium einen Namen gemacht, als er Brown in der Frage beriet, wie sich Großbritannien einer Euro-Mitgliedschaft entziehen könnte - dies zu einer Zeit, in der die Euro-Mitgliedschaft eine der Prioritäten von Blair zu sein schien. Die meisten Beobachter gingen davon aus, dass Cunliffe aufgrund seiner engen Verbindung zu Brown unter einer konservativ-dominierten Regierung nicht weiter aufsteigen würde. Doch Cameron hatte ihn gleich zu seinem Chefberater in EU-Angelegenheiten gemacht und ihn dann mit der Anweisung nach Brüssel entsandt, das britische Verhandlungsteam bei den Verhandlungen zu dem nächsten mehrjährigen Finanzrahmen mit harter Hand zu führen.

Eine weitere erwähnenswerte Berufung bei einer ansonsten routinemäßig verlaufenden Postenrotation der britischen Spitzendiplomaten war die Wahl von Simon Fraser als neuer Chef des Diplomatischen Dienstes in Großbritannien. Frasers Europaerfahrung stützt sich auf seine Zeit als Kabinettschef von Peter Mandelson, als dieser EU-Handelskommissar war. Als Brown Mandelson in sein Kabinett als Wirtschaftsminister zurückbeorderte, wurde Fraser ebenfalls zurückberufen und zum Staatssekretär in dessen Haus ernannt. Cunliffe und Fraser stehen symbolhaft für die Entschlossenheit von Cameron und Hague, einen Wandel des britischen außenpolitischen Entscheidungsprozesses einzuleiten - mit dem Ziel, die ,diplomatische' Macht des britischen Außenministeriums wieder herzustellen, britische Unternehmen besser zu unterstützen und den Schwerpunkt von der Europäischen Union zurück zur gesamten Welt und insbesondere hin zu den aufstrebenden Mächten zu verlagern. Diese Zielsetzung dürfte wohl den Ansichten von Clegg und seinen Liberaldemokraten widersprechen. Wahrscheinlich hat Clegg jedoch die weise Entscheidung getroffen, in diesen krisengeschüttelten Zeiten seine Aufmerksamkeit der britischen Wirtschaftspolitik zu widmen und den außenpolitischen Ambitionen Hagues gelassen entgegen zu sehen - unter der Voraussetzung, dass offene Feindseligkeiten gegenüber der Europäischen Union vermieden werden.

In Anbetracht notwendiger Mittelkürzungen im Außenministerium wie auch in anderen Ministerien verkündete Hague, dass er dennoch gewillt sei, in für die britische Wirtschaft bedeutsamen Regionen die britische Präsenz zu erhöhen. Damit meinte er insbesondere aufstrebende Mächte wie China, Indien, Brasilien und Russland. Mehr Personal in diesen Auslandsvertretungen soll durch eine signifikante Reduzierung des Personals, das für die Europäische Union oder die EU-Mitgliedstaaten zuständig ist, ermöglicht werden. Obwohl Hagues globale Ausrichtung in erster Linie auf handelspolitische und wirtschaftliche Interessen ausgerichtet war, schlug er doch kürzlich mit der Betonung auf der Wiederherstellung der traditionellen diplomatischen Stärken einen anderen Kurs ein. Er kündigte weitere Maßnahmen an, um das britische Außenministerium zu erneuern und so die Einflussmöglichkeiten Großbritanniens in Übersee langfristig zu verbessern. Erwähnenswert dabei ist, dass weder die Europäische Union noch ihre neuen, kürzlich umstrukturierten außen- und sicherheitspolitischen Verfahren in Hagues Vision erwähnt werden, Großbritannien wieder zu einem ernstzunehmenden diplomatischen Akteur zu machen. Hague schwärmte von weiteren 50 Personen für das AuBenministerium, die nach China entsandt werden, und 30 weiteren Personen für Indien. 
Von der Errichtung des Europäischen Auswärtigen Dienstes, dem Aufbau von EU-Botschaften oder der Entsendung von britischen Beamten in den Europäischen Auswärtigen Dienst war keine Rede. Vielmehr wurden die Bemühungen um eine Reduzierung des Budgets des Europäischen Auswärtigen Dienstes von den Briten angeführt. Lidington und andere Europaminister beteiligten sich daran und übten offen Kritik an den Forderungen von Ashton nach einer Erhöhung ihres sehr kleinen Budgets. Die britischen Minister verweigerten Ashton nicht nur ihre Unterstützung, sie boykottierten zudem ihre, bisher zugegebenermaßen bescheidenen Versuche, die ihr vom Europäischen Rat zugedachte Rolle auszuüben. Auch hier gab es nur wenig Protest von den Liberaldemokraten gegen diesen von der Koalition eingeschlagenen Kurs. Auffallend ist, dass Hague in seinen Reden Großbritannien und die britische Rolle in der Welt in den Mittelpunkt stellt - die Tatsache, dass Großbritannien ein EU-Mitgliedstaat und Hauptakteur der Gemeinsamen Außen-, Sicherheits- und Verteidigungspolitik der Europäischen Union ist, wird kaum erwähnt.

Aufgrund seiner Ablehnung des Politikstils von Blair und seinem Wunsch, die traditionelle Rolle des britischen Außenministeriums wiederherzustellen, erhielt Hagues Herangehensweise an die britische Außenpolitik allgemein große Zustimmung. Sie war aber auch Gegenstand eines mahnenden Leitartikels in der Financial Times. Diese schrieb, dass ,nun die Gefahr besteht, dass der Wunsch von Herrn Hague sich auf starke bilaterale Beziehungen zu den aufstrebenden Mächten des 21. Jahrhunderts zu konzentrieren, dazu führen könnte, dass er die traditionellen Beziehungen des Königreichs als weniger wichtig erachtet. Europa und die USA bleiben weiterhin entscheidend für den britischen Einfluss. " 30 Weiter heißt es dort, dass die Mittelumverteilung weg von diesen Regionen mit dem Ziel, die diplomatische Präsenz anderenorts zu stärken, die Tatsache nicht berücksichtigt, dass das Vereinigte Königreich für sich genommen in Indien und China wenig zählt - oder auch in Washington, wo die maßgebliche Rolle, die Großbritannien in der Europäischen Union einnimmt, seine Stellung untermauert. Nach Ansicht der Financial Times ist es also die volle Beteiligung Großbritanniens in der Europäischen Union, auf die es ankommt - die britische Haltung in der Krise der Eurozone und die unilateralen und bilateralen Reaktionen auf die Ereignisse in Libyen deuten allerdings nicht darauf hin, dass dies eine der Prioritäten der gegenwärtigen Regierung ist.

\section{Folgen für die Gemeinsame Sicherheits- und Verteidigungspolitik}

In den letzten Jahren kam die Gemeinsame Sicherheits- und Verteidigungspolitik (GSVP) der Europäischen Union zum Erliegen. Es gibt weder neue GSVP-Missionen noch ist es der Europäischen Verteidigungsagentur gelungen, den Markt für europäische Rüstungsgüter zu öffnen oder eine Rationalisierung der 27 verschiedenen nationalen Streitkräfte anzuregen. Die mangelnden Fortschritte lassen sich teilweise auch auf die Unwilligkeit Großbritanniens zurückführen - insbesondere der neuen konservativ-dominierten Koalitionsregierung -, diese Initiativen zu unterstützen. Stattdessen hat das Vereinigte Königreich engagiert den Kurs der Labour-Partei fortgesetzt, mit Frankreich alternativ eine bilaterale Initiative zur Verteidigungskooperation zu ergreifen. Premierminister Cameron und Präsident Sarkozy verkündeten bei einem Treffen im November 2010 ihre Absicht, diese auszubauen, und im April 2011 unterzeichneten die beiden Staaten den Vertrag über die Zusammenarbeit in der Verteidigungs- und Sicherheitspolitik. Mit diesem verpflichten sich die Regierungen in Paris und London unter anderem zu einer gemeinsamen Flugzeugträgerflotte, einer 10.000 Mann

30 Financial Times: Diplomacy makes a comeback... But Britain must not forget its traditional partners, Editorial, 09.09.2011. 
starken gemeinsamen Eingreiftruppe, der engen Kooperation bei Atom-U-Boot-Patrouillen, gemeinsamen Trainingsmaßnahmen und Einsätzen von Piloten sowie zur Entwicklung gemeinsamer Anlagen für Atomtests.

Kurz darauf, als es in der Europäischen Union zu einigen Unstimmigkeiten kam, übernahmen das Vereinigte Königreich und Frankreich die Führung - letztlich eher im NATOals im EU-Rahmen - bei der Militärintervention zur Unterstützung der Gegner des langjährigen libyschen Machthabers Muammar al-Gaddafi. In Großbritannien galt Cameron trotz der offensichtlichen Risiken eines solchen Unterfangens als ausgesprochen erfolgreich. Auch die bilateralen Beziehungen mit Frankreich im Verteidigungsbereich wurden von den Konservativen positiv aufgenommen, die ansonsten für ihre Abneigung gegenüber den Franzosen und ihre Ablehnung von Aktionen im EU-Rahmen bekannt sind. Das libysche Unternehmen (das von den Liberaldemokraten mitgetragen wurde) zeigte schlaglichtartig die Probleme, die die beiden Staaten haben, wenn sie sich zu Militäreinsätzen in Situationen entschließen, bei denen die USA nur verhalten agieren oder zögern, eine Führungsrolle zu übernehmen. Die Intervention in Libyen, für die sich Cameron vehement einsetzte, war eine Belastung für die britischen Streitkräfte bei ihren Einsätzen in Afghanistan und in Libyen, besonders nachdem die USA ihre Flugzeuge von den NATO-Einsätzen abgezogen hatten. ${ }^{31}$

Vor den Wahlen 2010 war bei den Konservativen noch von einem Rückzug des Vereinigten Königreichs aus der Europäischen Verteidigungsagentur die Rede. Dieses Vorhaben wurde jedoch nicht in die Koalitionsvereinbarung aufgenommen und bisher begnügte sich die Regierung mit einer Politik, die auf eine Begrenzung der Befugnisse und eine Reduzierung des Budgets der Agentur bedacht ist. Es ist unbestritten, dass - wie bei dem Abkommen von St. Malo - Großbritannien und Frankreich aus unterschiedlichen Beweggründen im Verteidigungsbereich zusammenarbeiten. Die Briten favorisieren eine Zusammenarbeit außerhalb des EU-Rahmens und sind bemüht, den USA ihre Bereitschaft zu signalisieren, weiterhin ihre militärischen Kapazitäten aufrechtzuerhalten und auch einzusetzen. Für die Franzosen ist die verteidigungspolitische Zusammenarbeit mit den Briten eine elementare Voraussetzung für den Erfolg der GSVP. Großbritannien und Frankreich kommen zusammen für 45 Prozent der Verteidigungsetats der Mitgliedstaaten der Europäischen Union auf (und wie anders würde es noch aussehen, wenn die deutschen Ausgaben dazu kommen würden!), für 55 Prozent der europäischen Einsatzkräfte und für 70 Prozent der Ausgaben für militärische Forschung und Technologie.

Wie Philip Stephens bemerkt hat, hat sich die Dreiecksbeziehung Großbritanniens mit Frankreich und Deutschland (dem Kern der Europäischen Union?) verkompliziert. ${ }^{32}$ Die Regierung von Cameron war bestrebt, gemeinsam mit Frankreich die Führung in Libyen zu übernehmen und die Eurozone mit all ihren Problemen Frankreich und Deutschland zu überlassen. Die Koalitionsregierung teilt eher mit Deutschland als mit Frankreich die Begeisterung für eine Verbesserung der EU-Wettbewerbsfähigkeit und die Gewährleistung eines funktionierenden Binnenmarkts. Mit beiden Staaten ist sie aber uneinig in der Frage eines türkischen EU-Beitritts sowie bei der EU-Finanzmarktregulierung, die sich ungünstig auf die City of London auswirken könnte. ${ }^{33}$ Einige Beobachter wünschen sich, dass Großbritannien die bilateralen britisch-französischen Beziehungen im Verteidigungsbereich um Deutschland erweitern würde und sich selbst in den bilateralen deutsch-französischen wirt-

31 Anand Menon: Two's company, but not enough: Reflections on Anglo-French defence cooperation, in: Adam Hug (Hrsg.): The New British Politics and Europe: Conflict or Cooperation?, London 2011, S. 30-34.

32 Philip Stephens: Europe's not so eternal triangles, in: Financial Times, 01.04.2011.

33 Bei dieser Frage bestehen allerdings Spannungen auch innerhalb der Koalition. 
schafts- und finanzpolitischen Beziehungen einbringen sollte. ${ }^{34}$ Im Vereinigten Königreich würde dies allerdings als ein Plus für die Europäische Union und als Verlust für Großbritannien betrachtet werden!

\section{Fazit: Cameron geht trotz Koalitionsregierung auf Distanz zur Europäischen Union}

Abschließend soll nicht unerwähnt werden, dass das Krisenmanagement der Europäischen Union im Umgang mit den Schwierigkeiten der Eurozone dazu führt, dass eine ganze Reihe von bekannten britischen EU-Berichterstattern gemeinsam ins Horn blasen und sich auf lange Sicht ausgesprochen pessimistisch zu den Überlebenschancen der Europäischen Union äußern. ${ }^{35}$ Martin Kettle zitiert Sir Stephen Wall, den ehemaligen britischen Ständigen Vertreter Großbritanniens bei der Europäischen Union und Chefberater in EU-Angelegenheiten von Blair und den wohl einflussreichsten pro-europäischen britischen Diplomaten der jüngsten Zeit, mit den Worten „Wir haben die EU auf der Höhe ihrer Zeit gesehen. Mit ein wenig Glück wird sie uns überleben, aber sie ist dabei sich zu verabschieden. Letzten Endes bestehen doch nur sehr wenige Institutionen für immer". ${ }^{66}$ Wie auch immer das Schicksal der Europäischen Union aussehen wird, die Krise der Eurozone hat die Koalitionsregierung veranlasst, sich von der Europäischen Union zu distanzieren. Cameron sprach im September 2011 vor dem kanadischen Parlament davon, dass es den Regierungschefs der Eurozone (das Vereinigte Königreich also nicht eingeschlossen) unbedingt gelingen muss, gemeinsam an einem Strang zu ziehen. Zur gleichen Zeit hat er sich auch mit anderen führenden Politikern außerhalb der Europäischen Union zusammengetan und einen Brief an den französischen Präsidenten Sarkozy in seiner Funktion als G20-Präsident übermittelt, der die Mitglieder der Eurozone dazu aufruft rasch zu handeln. Der Guardian sah in diesem Brief ,einen bedeutenden Wendepunkt der britischen Diplomatie, die in den letzten vier Jahrzehnten in der EU verankert war. Es [sei] beispiellos für einen britischen Premierminister gemeinsam mit Regierungschefs außerhalb der EU eine Warnung an die wichtigsten EU-Mitgliedstaaten auszusprechen“" ${ }^{37}$ Cameron ruft gewissermaßen die Mitglieder der Eurozone dazu auf, sich mit vollem Einsatz für eine engere politische und finanzpolitische Integration der Eurozone - aber nicht im Rest der Europäischen Union - einzusetzen. Betrachtet man dies in Verbindung mit der Entschlossenheit Camerons, das Vereinigte Königreich auf mittlere und längere Sicht außerhalb der Eurozone zu halten und bezieht die restriktive Natur des European Union Act mit ein, dann zeigt sich, dass der kurzfristig mäßigende Einfluss des liberaldemokratischen Koalitionspartners insgesamt von nur geringer Bedeutung ist. Der konservative Koalitionspartner wird bis zum Ende dieser Regierungsperiode deutliche Zeichen im britischen Verhältnis zur Europäischen Union gesetzt haben.

34 Stephens: Europe's not so eternal triangles, 2011.

35 Philip Stephens: Europe's return to Westphalia, in: Financial Times, 24.06.2011; Martin Kettle: The nationalists have won - Europe's dream is over, in: The Guardian, 24.06.2011; Paul Mason: The European Dream is in danger: prepare for another rude awakening, in: Financial Times, 25.05.2011; Peter Spiegel: European Integration is unravelling, in: Financial Times, 31.05.2011.

36 Kettle: The nationalists have won - Europe's dream is over, 2011.

37 The Guardian: PM tells Europe: Act now to save global economy, 23.09.2011. 